\title{
X-Ray Spectroscopy of Supernova Remnants
}

\author{
By R. PETRE
}

\author{
NASA/Goddard Space Flight Center, Code 660, Greenbelt, MD 20771, USA
}

\begin{abstract}
X-ray spectroscopy can provide vital information about the progenitors and environments of supernova remnants. Plasma diagnostics and spectral modelling can be used to infer the energy of the remnant, the density and composition of the surrounding medium, and the degree of equilibrium in the shock heated gas. A new generation of X-ray spectrometers, the first of which was the Broad-Band X-Ray Telescope (BBXRT), has improved our ability to make precise measurements of X-ray line fluxes and energies. We summarize the results obtained from the BBXRT mission. These include a definitive measurement of the $\mathrm{Fe} \mathrm{K}$ line centroid in the Tycho remnant, production of the first narrow-band X-ray maps (of Puppis A) and the first measurement of an electron-ion equipartition timescales in evolved remnants.
\end{abstract}

\section{Introduction}

Supernova remnants may be grouped into three broad categories, based on their X-ray and radio morphologies. The first of these shows shell-like structure in both bands. The $\mathrm{X}$-rays from these are thermal, arising from the shock heating of ejecta and interstellar material. Prominent examples of this class of remnant are Tycho and the Cygnus Loop. The second category shows centrally peaked emission in both bands; these are the plerions, or Crab-like remnants, after the class archetype. The X-ray emission is a non-thermal power law, dominated by synchrotron processes from the energetic electrons produced by the pulsar. A third category combines elements of the previous two. Some remnants, like Vela, possess both a thermal shell and a non-thermal synchrotron nebula, while others, like W44 and W28, have a shell-like radio structure combined with a center-filled X-ray morphology that nevertheless arises from thermal X-rays. This presentation focuses on remnants dominated by thermal X-ray emission, as we are primarily interested in what the plasma diagnostics can tell us about the supernova progenitor and the surrounding medium.

$\mathrm{X}$-ray observations, particularly spectroscopy, offer the most direct means of determining many fundamental properties of supernova remnants. The production of $\mathrm{X}$-rays is tied directly to the non-radiative shock heating of diffuse ISM material and ejecta by the expanding blast wave or the reverse shock, so measuring the temperature of the X-ray emitting gas offers the most direct measure of the shock velocity and hence the energy of the initial explosion. X-ray spectroscopy facilitates measurement of the abundances of $\mathrm{O}, \mathrm{Ne}, \mathrm{Mg}, \mathrm{Si}, \mathrm{S}$, and $\mathrm{Fe}$ in shock heated material, and thus allows a determination of how enriched it has become from SN nucleosynthesis products, or, in evolved remnants, the composition of the gaseous ISM. While optical measurements facilitate plasma diagnostics of radiatively cooling filaments, with densities typically $10^{4} \mathrm{~cm}^{-3}$ and a small filling factor in a typical ISM $(\leq 1 \%)$, X-ray spectroscopy allows density measurements of the much more prevalent diffuse ISM and low density clouds, typically with densities $0.1-10 \mathrm{~cm}^{-3}$. Most recently, spatially resolved X-ray spectroscopy has allowed us to examine the variation of density and abundances across and around remnants, which can ultimately lead to inferences regarding the symmetry of the explosion and the pre- and post-shock conditions in the ISM.

The X-ray spectra of thermal supernova remnants are dominated by strong line emission from highly ionized nucleosysnthesis products. The $\mathrm{X}$-ray band includes $\mathrm{K}$ shell 
TABLE 1. Prominent X-ray Lines from Supernova Remnants

\begin{tabular}{lcc}
\hline \hline Element & $\begin{array}{c}\text { He- } \alpha \text { transition } \\
\text { energy (keV) } \\
\text { average of triplet) }\end{array}$ & $\begin{array}{c}\text { Ly- } \alpha \text { transition } \\
\text { energy (keV) }\end{array}$ \\
\hline Oxygen & $\mathbf{0 . 5 7 0}$ & $\mathbf{0 . 6 5 3}$ \\
Neon & $\mathbf{0 . 9 1 6}$ & $\mathbf{1 . 0 1 6}$ \\
Magnesium & $\mathbf{1 . 3 4}$ & 1.476 \\
Silicon & $\mathbf{1 . 8 6}$ & 2.006 \\
Sulfur & $\mathbf{2 . 4 6}$ & 2.622 \\
Argon & $\mathbf{3 . 1 4}$ & 3.315 \\
Calcium & $\mathbf{3 . 9 0}$ & 4.093 \\
Iron & 6.70 & 6.970 \\
Iron L blend & $\mathbf{0 . 6 8 - 1 . 1 5}$ & \\
\hline
\end{tabular}

Bold face indicates strongest lines.

emission lines from carbon through nickel; the most prominent lines, as listed in Table 1 are from $\mathrm{O}, \mathrm{Ne}, \mathrm{Mg}, \mathrm{Si}, \mathrm{S}$ and $\mathrm{Fe}$. In addition, the $\mathrm{Fe} \mathrm{L}$ band around $1 \mathrm{keV}$ is covered. As these lines originate from a recently shock- heated, low density $\left(\sim 1 \mathrm{~cm}^{-3}\right)$ plasma, it is almost certain that ionization equilibrium has not been attained. The non-equilibrium effects can be pronounced. For instance, in a $10^{7} \mathrm{~K}$ plasma in thermal equilibrium, one would find fully stripped $\mathrm{Mg}$ ions, $\mathrm{S}$ ions with one remaining electron, and $\mathrm{Si}$ ions with two electrons. Yet for most SNR the dominant line from all these elements is the 2-1 transition of the helium-like (two-electron) state. Thus fitting to a coronal plasma model (e.g., Raymond \& Smith, 1977) will yield too low a temperature. Additionally, these lines tend to be much stronger than in equilibrium, leading to the incorrect conclusion that the abundances of these metals are substantially enhanced (e.g., Berker et al., 1979, 1980). The only mechanism for ionizing the atoms in the low density plasma is collisions with electrons. These collisions are so infrequent that a substantial amount of time is required for the ionization state of the gas to approach that expected for the temperature of the ions. An upwardly ionizing gas will spend a shorter or longer amount of time in a given ionization state, depending on the ionization potential to the next state. In particular, since the ionization potential of an He-like ion to its $\mathrm{H}$-like state is high, it spends a relatively long time in the He-like state. The net result is that the He-like lines dominate SNR X-ray spectra. The absolute timescale for the upward ionization can be parameterized by either $n t$, where $n$ is the preshock density and $t$ is the age, or $\eta=n^{2} E$, where $E$ is the thermal energy associated with the explosion (typically $10^{51} \mathrm{erg}$ ).

In addition to the line emission, there is also a continuum component arising primarily from bremsstrahlung radiation. Its shape is dependent on the degree of thermal equipartition between the ions and electrons. The presence of this continuum underlying the line emission must be taken into account when line strengths are inferred.

It is only in recent years that spectrometers with sufficient spectral resolution, collecting area and bandpass have made it possible to take into account the possibly complicated conditions in SNR in inferring properties. The first instruments to offer sufficient spectral resolution were the Einstein SSS and FPCS. While these clearly resolved many lines and allowed the first true plasma diagnostics to be performed, the SSS suffered from insufficient resolution to distinguish, e.g., the He-like line from the $\mathrm{H}$-like line in $\mathrm{Si}$, and the 
FPCS was limited by its small collecting area to observing only the very brightest remnants. However, the FPCS and SSS clearly established the ubiquity of non-equilibrium conditions in SNR (Hamilton, Sarazin \& Szymkowiak, 1986a; b; Winkler et al., 1982; Hwang et al. 1993). The most recent generation of X-ray spectrometers has overcome these shortcomings and offer the added dimension of spatial resolution. These include the Broad-Band X-Ray Telescope (BBXRT) and the spectrometers on the ASCA satellite. As the ASCA results are still in a preliminary state and the first data are presented elsewhere in these proceedings, this paper will focus on the more thoroughly analyzed BBXRT data.

The BBXRT was the first instrument to offer high-throughput, modest resolution Xray spectroscopy across the $0.3-10 \mathrm{keV}$ band, allowing the first simultaneous spectroscopy of all $\mathrm{K}$ lines from $\mathrm{O}$ through $\mathrm{Ni}$. It had a spectral resolution of $\sim 100 \mathrm{eV}$ at $1 \mathrm{keV}$ and $\sim 155 \mathrm{eV}$ at $7 \mathrm{keV}$. Its focal plane spectrometers had five discrete detection elements, making possible crude spatially resolved spectroscopy. A more complete description can be found in Serlemitsos et al. (1993). The BBXRT was flown as part of the 9-day Astro-1 mission on the Space Shuttle Columbia. Of the more than 50 targets it observed, eight were supernova remnants (in addition to $\mathrm{SN1987a).} \mathrm{The} \mathrm{main} \mathrm{results} \mathrm{of} \mathrm{these}$ observations are summarized below.

Because of its broad bandpass and moderate spectral resolution, the BBXRT facilitates possible the use of either of the two most often employed spectral analysis techniques: line diagnostics and broad-band model fitting. We make use of both below; in general the results yielded by the two are identical. We compare our data with the grid of non-time-dependent ionization models by Hamilton, Sarazin \& Chevalier (1983), which model the development of X-ray lines in a Sedov blast wave. The Sedov solution formally describes self-similar adiabatic expansion of a blast wave from a point explosion in a cold, uniform medium. Work by Itoh (1977) and others have shown that an adiabatically expanding blast wave relaxes toward a Sedov solution when the mass of swept-up ISM exceeds $\sim 10$ times the mass of the SN ejecta. The Sedov solution can be completely characterized by three parameters corresponding to suitable choices of mass, length and time. A typical combination of such parameters is the age $t$ of the remnant, its energy $E$ and the ambient interstellar hydrogen density $n$. However, when modelling the Xray spectrum, using either a coronal equilibrium or a non-equilibrium ionization (NEI) model, the spectral shape is characterized by only two parameters. The parameters used by Hamilton, Sarazin \& Chevalier in creating their grid of NEI models are $T_{s}$, the temperature of the shock, and $\eta=n^{2} E$. The parameter grid covered by their models, and the relationship of $T_{s}$ and $\eta$ to other parameters, is shown in Fig. 4. One parameter of note is the product $n t$, where $t$ is the remnant age. The larger the $n t$ product, (towards high $\eta$ and low $T_{s}$ ), the closer the plasma approaches ionization equilibrium.

No observed supernova remnant is completely described by the Sedov solution: many of the best studied remnants are too young to have swept up sufficient mass, and most if not all remnants have encountered ISM inhomogeneities, both small and large. However, Hamilton \& Sarazin (1984) have shown that for SNR expanding into non-uniform media, and for young SNR which have not yet swept up the requisite ISM mass to physically approximate a Sedov solution, the $\mathrm{X}$-ray spectrum can be modeled by using two or more NEI components.

\section{Historical SNR - Tycho and Cas A}

Tycho, the remnant of a Type Ia supernova, and Cas A, the remnant of a Type II, are the most extensively studied thermal SNR in X-rays. This is because they are bright and 
hot, thus accessible by virtually any X-ray observatory, and display strong Fe $\mathrm{K}$ lines. As they are both younger than $450 \mathrm{y}$, their X-ray spectra are dominated by ejecta, and the $\mathrm{X}$-ray spectra therefore allow us to make inferences about the nature of the progenitor star.

For Tycho, the current best model of the X-ray spectrum, incorporating data from a number of instruments to cover the $0.1-20 \mathrm{keV}$ band, invokes two time-dependent ionization components, one representing the propagating blast wave, and the other a reverse shock encountering layered ejecta (Hamilton, Sarazin and Szymkowiak, 1986b). With the BBXRT it was possible for the first time to observe simultaneously the high and low energy components and thus remove any systematic uncertainties associated with instrumental cross calibrations, and to seek detailed spectral differences around the remnant. A BBXRT spectrum is compared with the spectrum from a much longer Einstein SSS exposure in Fig. 1. Two BBXRT spectra, of opposite sides of the remnant, yield identical line strengths to $\leq 5 \%$ despite the fact that they sample emission from regions separated by a distance of approximately $6 \mathrm{pc}$. This provides the first X-ray constraint on the azimuthal symmetry of a supernova explosion and suggests that the medium into which Tycho is expanding is also uniform to $\sim 5 \%$. Despite the limited number of counts in the BBXRT spectrum, a number of strong lines are visible to the eye, including some that were previously never resolved (for example the "beta" transition of Si XIV at $2.01 \mathrm{keV}$ ). Merely measuring the energy centroids and the intensity ratios of the prominent lines provides some new and interesting information. For instance, one source of controversy based on the results of lower resolution spectrometers has been the centroid energy of the Fe K line (Hamilton, Sarazin \& Szymkowiak, 1986b). The BBXRT measures the centroid directly, without the need for accounting for the slope of the underlying continuum. The measured value of $6.41 \pm 0.02 \mathrm{keV}$ indicates a very low ionization state (Fe XIX or lower).

We have used the diagnostics for non-equilibrium plasmas from Hamilton, Sarazin, $\&$ Chevalier (1983) to constrain the parameters characterizing the plasma, $T_{s}$ and $\eta$. The parameter values allowed by these diagnostics using the lighter elements $(\mathrm{Mg}, \mathrm{Si}$ and S) are disjoint from those allowed by the Fe K line energy. We conclude that the environment in which the $\mathrm{Fe}$ is embedded, presumably the innermost shell of ejecta, is hotter and less dense than the other shells. While the possibility of this was suggested by Hamilton, Sarazin \& Szymkowiak (1986b), the BBXRT provides a direct measurement almost trivially.

While the Cas A spectrum in Fig. 2 displays the same general properties as that of Tycho, namely strong lines from $\mathrm{Mg}, \mathrm{Si}, \mathrm{S}$, and $\mathrm{Fe}$, the line shapes and relative strengths are different, as is the strength of the high energy continuum. In particular, the Si line is not as prominent as in Tycho, the Fe $\mathrm{K}$ emission is substantially stronger, and all lines are intrinsically broadened. Markert et al. (1983) ascribed the shape of the Si line to Doppler broadening from an expanding ring of ejecta observed at a range of velocities. In contrast to the $\mathrm{Fe} \mathrm{K}$ line in Tycho, the centroid energy in Cas $\mathrm{A}$ is around $6.6 \mathrm{keV}$, more consistent with mostly stripped Fe. Also, the BBXRT reveals for the first time that its shape is inconsistent with a single, narrow line. It can be fit with a single broad line at $6.6 \mathrm{keV}$ of intrinsic width $84 \mathrm{eV}$, or as two narrow lines with energies 6.46 and $6.65 \mathrm{keV}$. If we assume a single, broad line, then the centroid is larger than any value on the grid of models for a single Sedov-Taylor blast wave constructed by Hamilton, Sarazin, \& Chevalier (1983). This suggests the need to model Cas A as the sum of multiple non-equilibrium models, as was done for Tycho. 
Tycho SNR

BBXRT and SSS
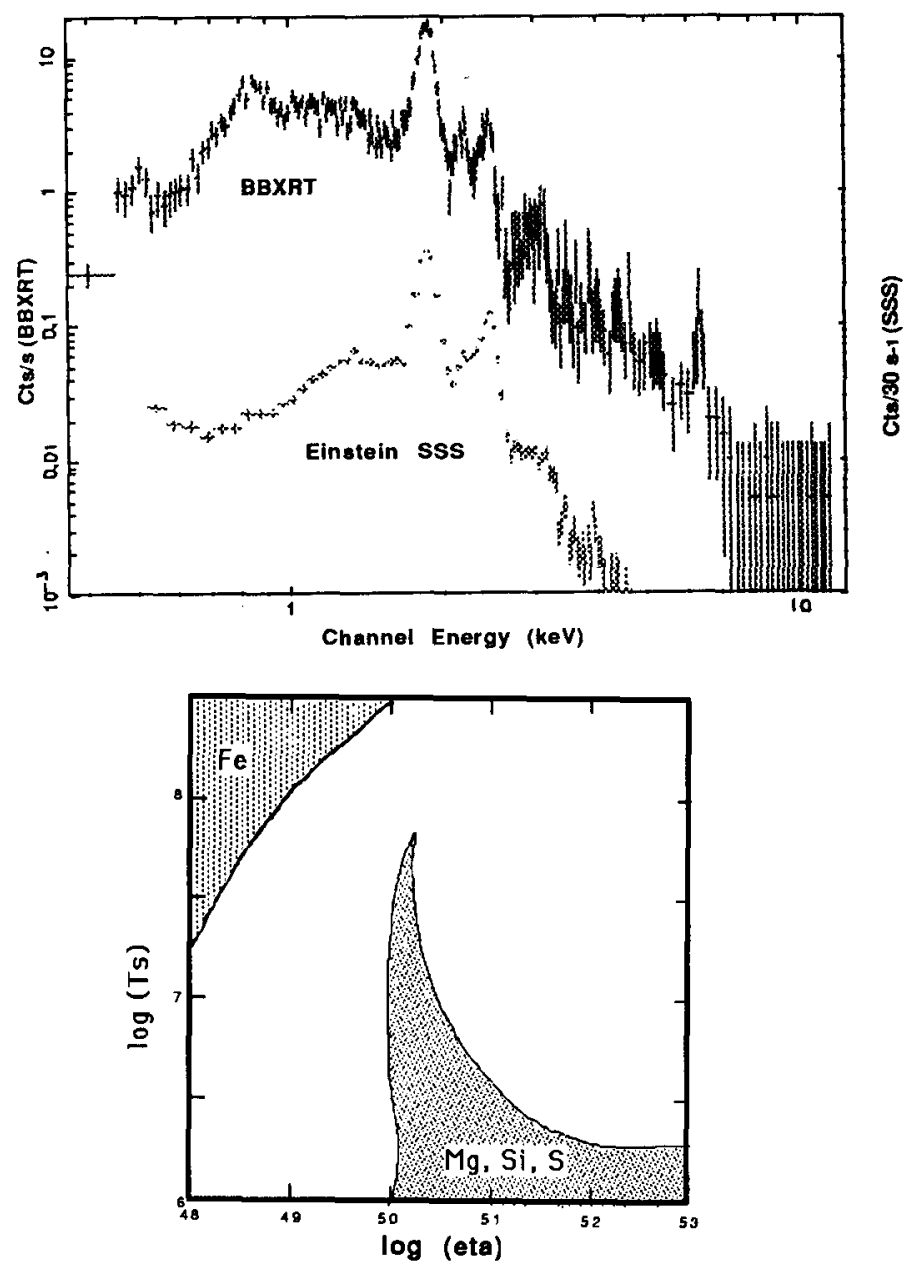

FIGURE 1. (upper). BBXRT spectrum of Tycho SNR (top trace), compared with Einstein SSS spectrum (bottom trace). (lower). Allowed 90 percent confidence regions of $T_{s}$ and $\eta$ for Tycho. Allowed regions for Fe $\mathrm{K}$ diagnostics and those for lighter elements are completely disjoint.

\section{Evolved SNR}

For evolved supernova remnants, whose $\mathrm{X}$-ray emission is dominated by swept up ISM material instead of ejecta, $\mathrm{X}$-ray spectroscopy reveals more information about the ISM and shock physics than about the progenitor and the supernova event. The BBXRT observed all or part of five evolved SNR: IC 443, CTB109, Vela, N132D, and Puppis A. Below we briefly discuss our findings on each of these.

The global morphology of the evolved remnant IC 443 has been strongly influenced by the presence of a molecular cloud in the line of sight (Petre et al., 1988). Bright X-ray emission, relatively unobscured by the molecular cloud, arises in a region of enhanced density in the vicinity of (but not associated with) bright optical filamentation in the northern portion of the remnant. In Fig. 3 we show spectra from the edge of the shell and the interior of the bright knot, about $6 \mathrm{pc}$ behind the shell. Spectral differences are 


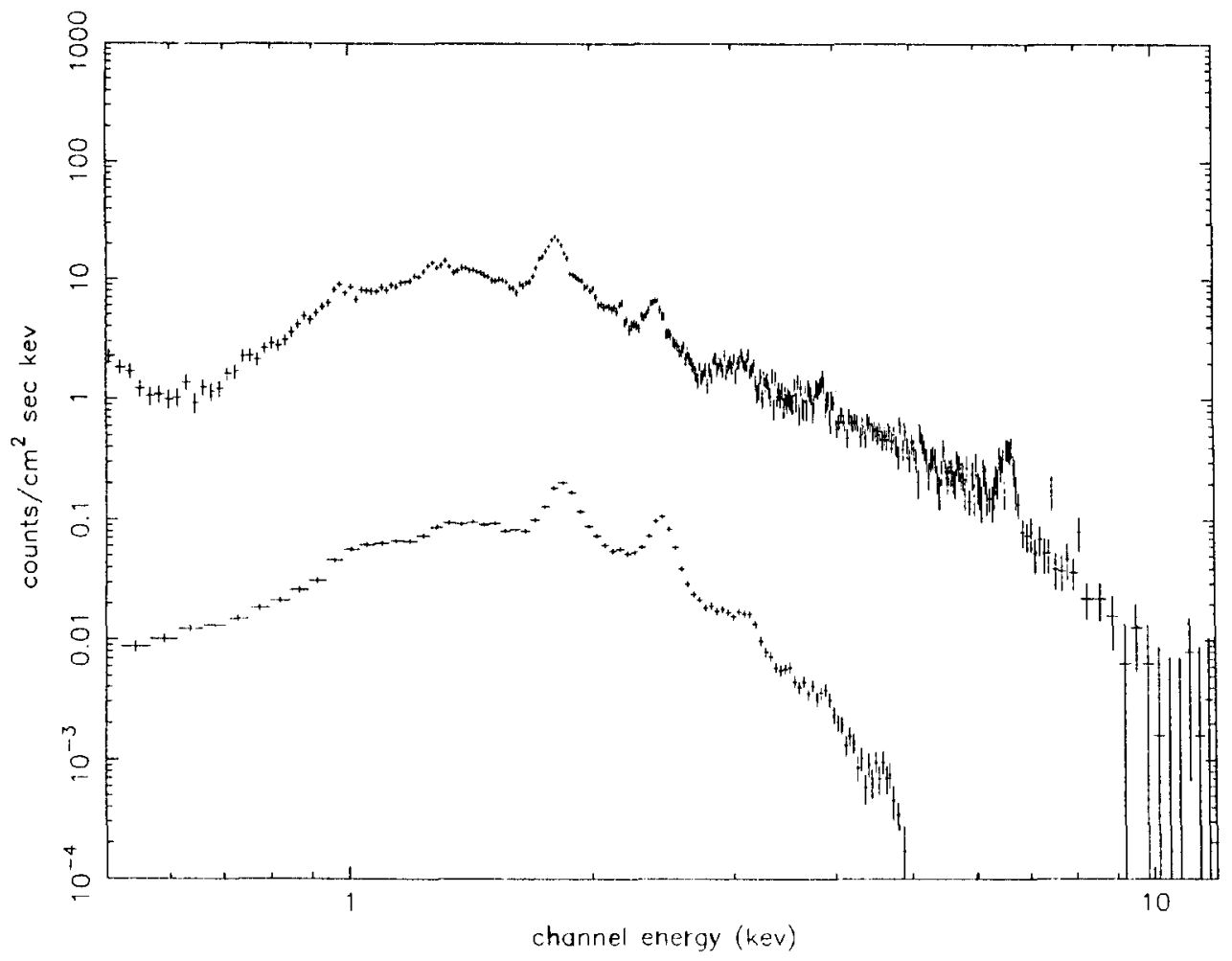

FIgURE 2. (top). BBXRT spectrum of Cas A. (top trace), compared with Einstein SSS spectrum (bottom trace). (bottom) Fe $\mathrm{K}$ region of Cas A spectrum. Line is fit using two narrow components, at 6.46 and $6.65 \mathrm{keV}$.

quite apparent. There is a clear trend with increasing distance behind the shock towards lines of higher ionization state in the band around $1 \mathrm{keV}$ (a band dominated by Ne IX, $\mathrm{Ne} \mathrm{X}$, and the $\mathrm{Fe} \mathrm{L}$ blend), the $\mathrm{Mg} \mathrm{K}$ region around $1.35 \mathrm{keV}$ and the $\mathrm{Si} \mathrm{K}$ band around $1.80 \mathrm{keV}$.

Compared with the spectra of the younger remnants, the lines from IC 443 are not nearly as strong. In fact, model fitting suggests that all the elements from $\mathrm{Ne}$ through Fe are underabundant with respect to solar (see Table 2). Despite the relatively weak line emission, the spectral resolution and broad bandpass of the BBXRT facilitate for the first time on this remnant the kind of plasma diagnostics performed previously on only the bright, young remnants. In Fig. 4 we show the location in the $\eta-T_{s}$ plane of the best-fit non-equilibrium model. The primary difference between the rim and the interior is that at the rim a model in which electron-ion equipartition has not been reached $\left(T_{i} \neq T_{e}\right)$ is preferred, while in the interior, an equipartition model is preferred. This is the first measurement of an equipartition timescale in a supernova remnant: from Sedov dynamics, we estimate an equipartition time of $\sim 1,000 \mathrm{y}$. The allowed values of $\eta$ and 

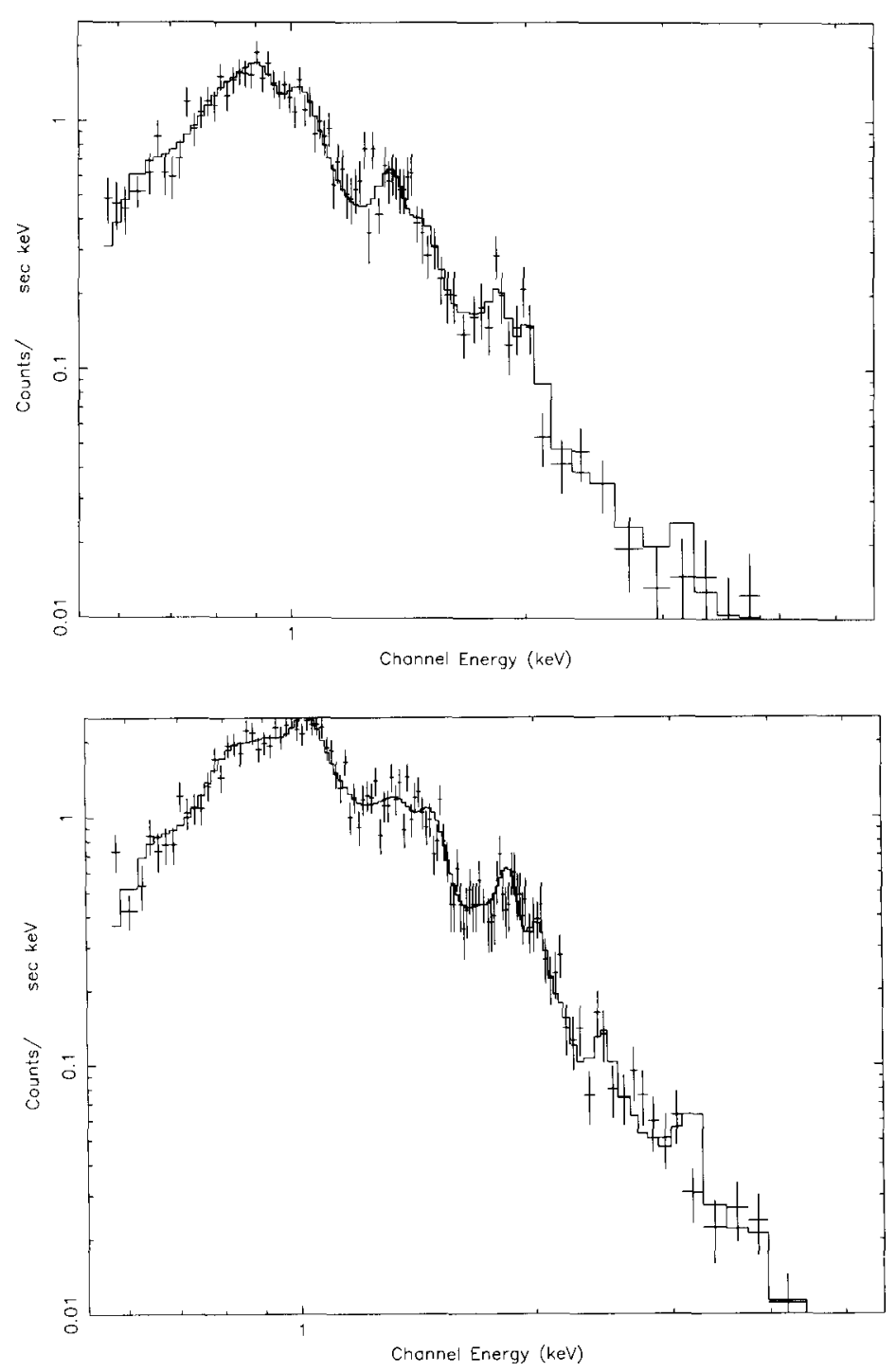

FIGURE 3. Spectra from the rim (top) and interior (bottom) of the X-ray bright knot in IC 443. Changes in the spectrum around $1 \mathrm{keV}$ and $1.85 \mathrm{keV}$ are readily apparent.

$T_{s}$ are in a region where the differences between non-equilibrium and equilibrium models becomes small, suggesting a rather evolved remnant.

The remnant CTB109 (G109.1-1.0) is unique among galactic supernova remnants: in addition to a well-defined semicircular shell, it contains a centrally situated binary X-ray pulsar, 1E2259+586 (Gregory \& Fahlman, 1979; Fahlman \& Gregory, 1980). The angular extent is sufficient so that BBXRT could simultaneously obtain spectra of both the diffuse emission and the pulsar in different detector elements. The BBXRT observation represents the first high quality spectrum ever obtained of CTB109. The spectrum displays prominently the $\mathrm{He} \alpha$ lines of $\mathrm{Ne}, \mathrm{Mg}$, and Si. Fitting it with a single NEI model has turned out to be quite problematic. The Ne IX/Ne X ratio is very large, suggesting 


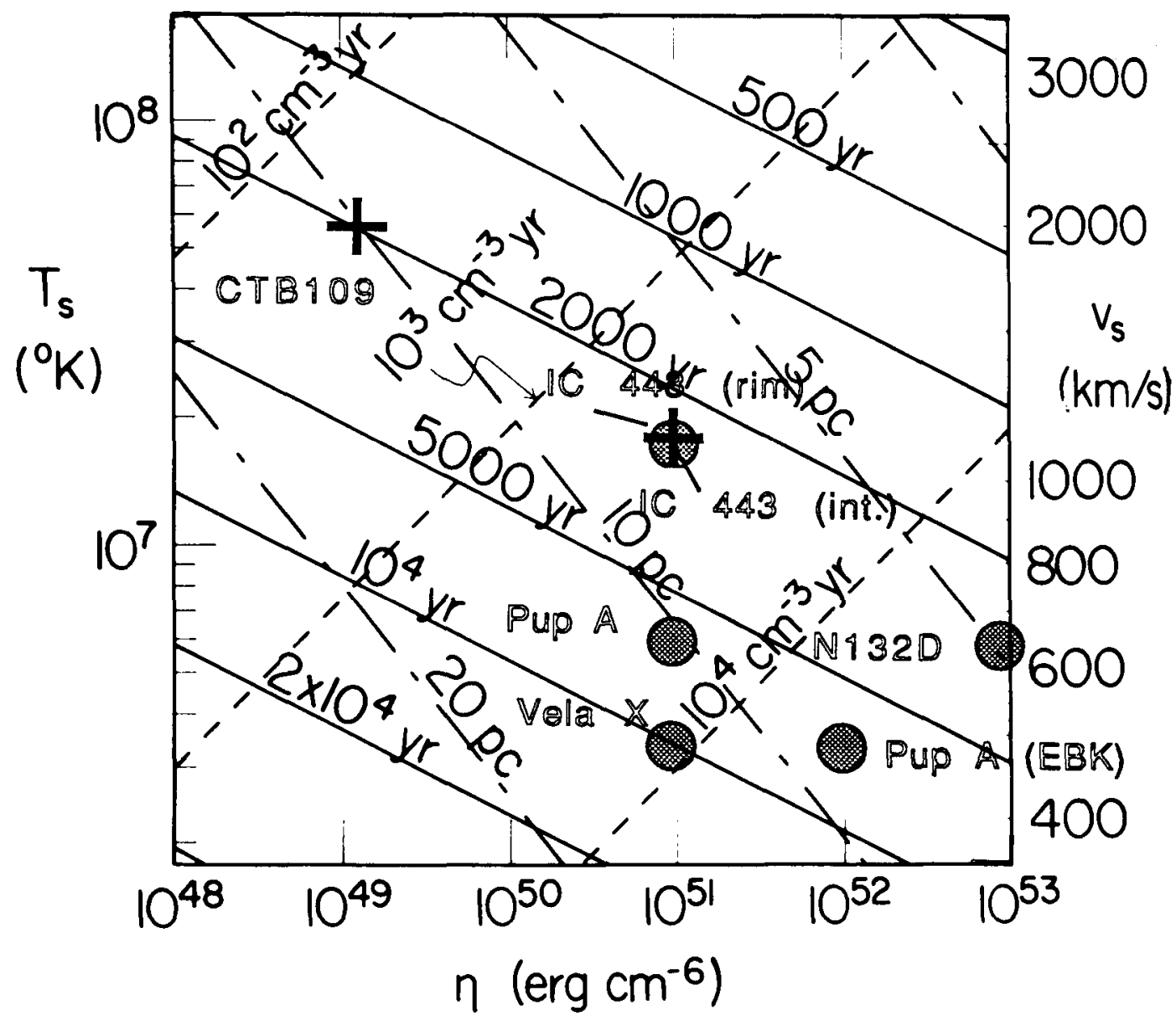

Figure 4. Best fit solutions for evolved remnants observed by the BBXRT, displayed in $\eta-T_{s}$ plane from Hamilton, Sarazin \& Chevalier (1983). Filled circles are for fits where $T_{i} \neq T_{e}$, and plus signs represent fits for which $T_{i}=T_{e}$

either a low temperature or small $n t$ product. At the same time, the continuum above $2 \mathrm{keV}$ is strong, requiring a high shock temperature. While this might suggest the presence of two NEI components, the BBXRT data are well fit by a single component model, with $T_{s}=5 \times 10^{7} \mathrm{~K}, \eta=10^{49} \mathrm{erg} \mathrm{cm}{ }^{-6}, n t=300 \mathrm{~cm}^{-3} \mathrm{y}$, and $T_{i}=T_{e}$. The approximate time since the shock passed this region is $\sim 2,000 \mathrm{y}$, comparable with the equipartition time estimated for IC 443. Another interesting result is the large abundances of oxygen and neon, and the low abundance of iron, required by the fits (see Table 2). If we are observing ejecta, this offers strong evidence that CTB109 in the remnant of a Type II supernova. It is interesting that the both spectra for which an ion-electron equipartition models is preferred sample remnant interiors. Vela $\mathrm{X}$ is a very complex region of the Vela supernova remnant containing the Vela pulsar and its associated synchrotron nebula. Despite the dominance of the hard non-thermal component, it was possible using the BBXRT to measure the temperature of the hot gas in the Vela $\mathrm{X}$ region. Our best fit model yields $T_{s}=3 \times 10^{6} \mathrm{~K}$ and $\eta=10^{51} \mathrm{erg} \mathrm{cm}^{-6}$. Again, the relatively high $n t$ product, $10^{4} \mathrm{~cm}^{-3} \mathrm{y}$, suggests that the gas is approaching ionization equilibrium. Even in the interior of this very evolved SNR, we find $T_{i} \neq T_{e}$. The relatively small number 
TABLE 2. Abundance Measurements of Evolved SNR using BBXRT

\begin{tabular}{lcccc}
\hline Remnant & CNO/Si & $\mathrm{Ne} / \mathrm{Si}$ & $(\mathrm{Fe}+\mathrm{Ni}) / \mathrm{Si}$ & $\mathrm{Si} /$ solar \\
\hline IC 443 (rim) & 0.5 & 0.3 & 1.0 & 0.2 \\
IC 443 (interior) & 0.4 & 0.5 & 1.8 & 0.1 \\
CTB109 & 3.5 & 1.5 & 0.0 & 2.4 \\
Vela X & 1.0 & 1.0 & 1.0 & 1.0 \\
N132D & 0.7 & 0.6 & 0.9 & 0.1 \\
Puppis A (E. knot) & 0.6 & 0.8 & 0.6 & 1.0 \\
Puppis A (composite) & 0.7 & 0.9 & 0.9 & 0.6 \\
\hline
\end{tabular}

of counts in the spectrum precludes any statement about abundances, except that they are not substantially different from solar.

N132D, the most X-ray luminous SNR in the Large Magellanic Cloud, is thought to be the remnant of a Type II explosion, based on its oxygen-rich optical spectrum (Lasker, 1978). Despite the very brief BBXRT exposure ( $475 \mathrm{~s}$ ), we derive a unique solution from model fitting. The best fit NEI model has $T_{s}=5 \times 10^{6} \mathrm{~K}, \eta=10^{52} \mathrm{erg} \mathrm{cm}^{-6}$, and $T_{i} \neq T_{e}$. The large $n t$ product $\left(3 \times 10^{4} \mathrm{~cm}^{-3} \mathrm{y}\right)$ indicates near-equilibrium conditions. The lack of strong line emission above $1 \mathrm{keV}$ is apparent. This is due to reduced abundances of $\mathrm{Mg}$, $\mathrm{Si}$ and Fe. To compare our results with those from the Einstein FPCS (Hwang et al., 1993), we explicitly incorporated into our fitting the strong lines of O VII, and O VIII. The ratio between these lines is only marginally consistent with the FPCS result. There is also a statistically significant detection of a line at $500 \mathrm{eV}$, consistent with N VII Ly $\alpha$.

While the BBXRT was unmatched in its combination of spectral resolution, sensitivity and bandpass, its observation of Puppis A offers something truly unique. The BBXRT performed a scanning observation across Puppis A, covering virtually the entire remnant. Attitude reconstruction has been performed on these data, yielding a map of the entire remnant with $\sim 6$ arc-minute resolution. Taking advantage of BBXRT's spectral resolution, we have created maps containing only those events which appear in the various lines, thus producing the first ever narrow-band X-ray images of a supernova remnant (or any cosmic X-ray source). These maps offer a new technique for studying how the abundances and ionization structures vary across a supernova remnant. Figs. 5 and 6 provide examples of the promise of this technique. In Fig. 5, we show the flux ratio between $\mathrm{O}$ VII and O VIII, and in Fig. 6, the flux ratio between Ne IX and Ne X. There is clearly structure in both ratios across the remnant, indicating variations either in temperature or ionization state. More interestingly, there are clear differences between the two ratios, which indicate clear differences in ionization state across the remnant. As there maps are preliminary, we draw no strong inferences from them, but merely point out the potential usefulness of narrow band X-ray images as diagnostics. We have also carried out a more conventional analysis of two spectra from Puppis A, a composite spectrum and a spectrum of the brightest X-ray feature in the remnant, the eastern bright knot (Petre et al., 1982). We find both spectra require $T_{i} \neq T_{e}$, and in Fig. 4, the two can be seen to lie along a line of constant age. The primary difference is that due to its higher density the eastern bright knot has a higher $n t$ product than the remnant as a whole. 


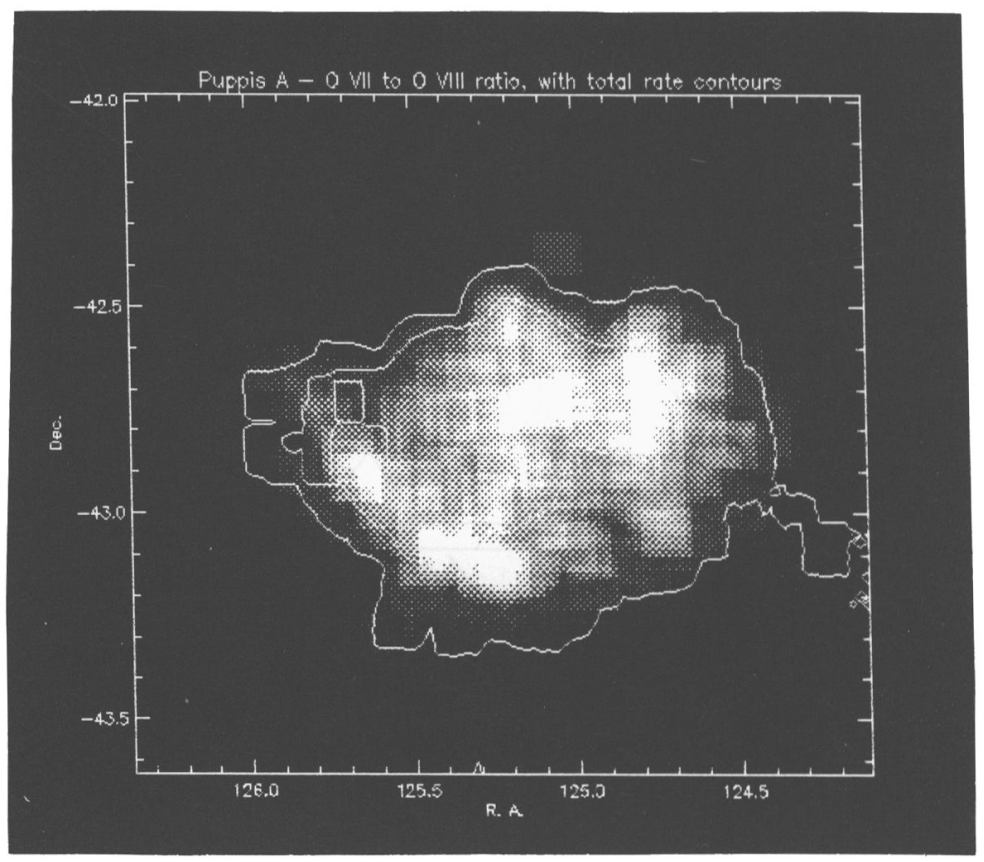

Figure 5. O VII to O VIII line ratio in Puppis A, overlaid on surface brightness contours.

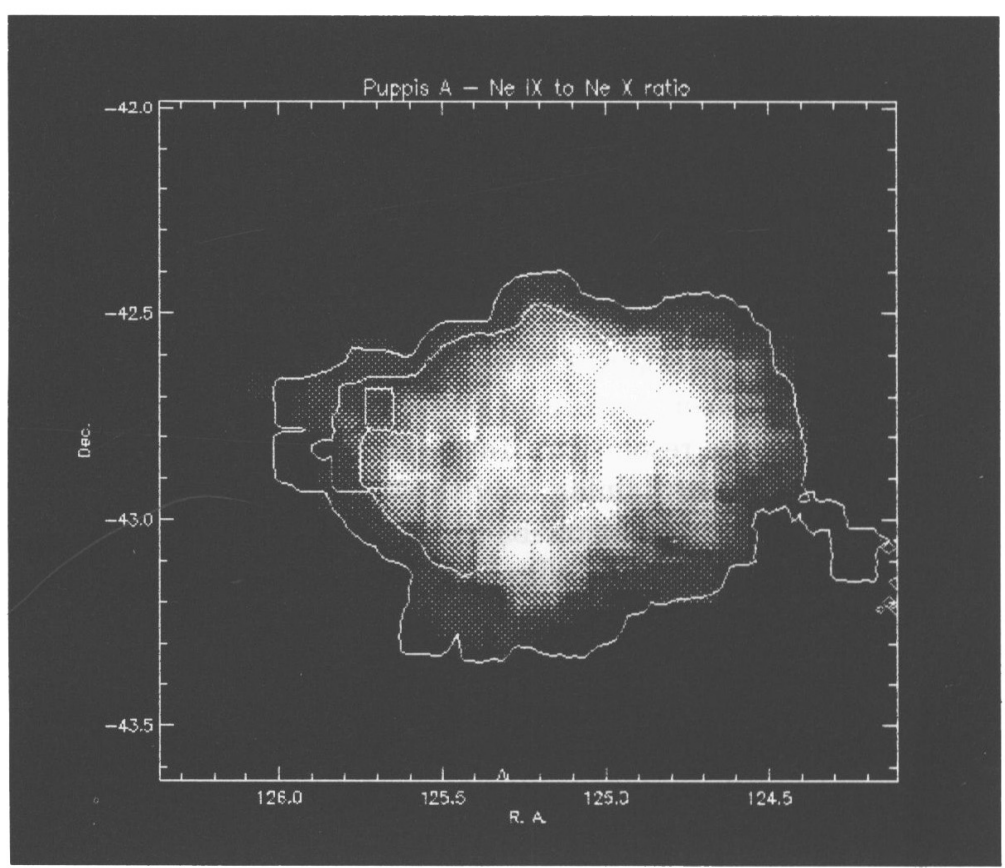

Figure 6. Ne IX to Ne X flux in Puppis A, overlaid on surface brightness contours. Comparison with $\mathrm{O}$ ratio indicates differences in ionization structure. 


\section{Summary}

With the BBXRT and its successors, X-ray spectroscopy of SNRs has entered a new era. No longer can the starting point of spectral analysis be fitting a one- or two-temperature coronal plasma model to the data. As the spectra of Cas A and Tycho demonstrate, even a single component NEI model is insufficient. Our ability to refine information regarding progenitor properties will become even more dependent on the input models and the degree of certainty of the atomic physics line strength calculations.

For the evolved supernova remnants, the data and our knowledge have not yet reached such a critical juncture; we have much to learn from our first application of NEI models to these data. In Fig. 4 and Table 2, we have summarized the BBXRT fit results for the evolved remnants. Fig. 4 shows the range of physical conditions found in SNR, from extreme nonequilibrium in the interior of CTB109, to near equilibrium in N132D. It also shows that $T_{i} \neq T_{e}$ is found in many locations, but not everywhere. In particular, we find electron-ion equipartition only in the interiors of these remnants (though not all interiors show equipartition). In Table 2, we have compiled the best-fit abundance values, scaled to that of $\mathrm{Si}$, plus the absolute value of the Si abundance compared with the cosmic value. The only general conclusion that can be reached is that abundances vary strongly from remnant to remnant. Reaching more general conclusions about whether the abundances within the hot gas also vary strongly across remnants, and whether the suggestion that electron-ion equilibration times can be inferred in many remnants, requires a more comprehensive program of spatially resolved X-ray spectroscopy. That capability is currently available using the newly-launched Japanese/US observatory ASCA.

The author would like to gratefully acknowledge the many individuals whose efforts made possible the BBXRT mission and the results presented here. P. Serlemitsos, the BBXRT Principal Investigator, is the prime mover behind the entire effort. A. Szymkowiak has performed the analysis of the Cas A data, and together with K. Arnaud and J.-H. Rho made the Hamilton NEI models available in XSPEC. A. Smale is responsible for the BBXRT data extraction software and $\mathrm{K}$. Weaver played the key role in development of the BBXRT spectral response matrices. Finally S. Snowden helped the author overcome the needless complexities of TeX.

\section{REFERENCES}

Becker, R. H., et al. 1979, ApJL, 246, L73-6.

Becker, R. H., et al. 1980, ApJL, 235, L5-8.

Fahlman, G. G., \& Gregory, P. C. 1981, Nature, 293, 202-4.

Gregory, P. C., \&Fahlman, G. G. 1980, Nature, 287, 805- 7.

Hamilton, A. J. S., Sarazin, C. L., \& Chevalier, R. A., 1983, ApJS, 51, 115-48.

Hamilton, A. J. S., Sarazin, C. L. 1984, ApJ 284, 601-11.

Hamilton, A. J. S., Sarazin, C. L., \& Szymkowiak, A. E. 1986a, ApJ, 300, 698-712.

Hamilton, A. J. S., Sarazin, C. L., \& Szymkowiak, A. E. 1986b, ApJ, 300, 713-21.

Hwang, U., Hughes,J. P., Canizares, C. R.,\& Markert, T. H. 1993, ApJ, 414, 219.

Itoh, H. 1979, Pub. Astr. Soc. Japan, 29, 813-27.

Lasker, B. M. 1978, ApJ, 223, 109-21.

Markert, T. H., Canizares, C. R., Clark, G. W., \& Winkler, P. F., 1983, ApJ, 268, 134-44.

Petre, R., Szymkowiak, A. E., Seward, F. D., \& Willingale, R. 1988, ApJ, 335, 215-38.

Raymond, J. C. \& Smith, B. W. 1977, ApJS, 35, 419-66. 
Serlemitsos, P. et al., 1992, Frontiers of X-Ray Astronomy, ed. Y. Tanaka and K. Koyama, pp. 221- 231. Tokyo: Universal Academy Press.

Winkler, P. F., Canizares, C. R., Clark, G. W., Markert, T. H., Kalata, K., \& Schnopper, H. W. 1982, ApJL, 246, L27-32. 